$\mathbb{T}$ periodica polytechnica

Mechanical Engineering

$53 / 2(2009) 55,60$

doi: 10.3311/pp.me.2009-2.01

web: http://www.pp.bme.hu/me

(c) Periodica Polytechnica 2009

RESEARCH ARTICLE

\section{Experimental and numerical study on cavitating vortex shedding behind a square cylinder}

\author{
Ferenc Hegedûs / Roland Rákos / László Kullmann \\ Received 2010-01-18
}

\begin{abstract}
Comparison between experimental and numerical results of the cavitating vortex shedding behind a square cylinder is presented. The side length of the experimental and numerical model was $25 \mathrm{~mm}$ and $20 \mathrm{~mm}$, respectively. The water velocity in the $0.2 \times 0.05 \mathrm{~m}$ test section of the channel was varied between 1 and $12 \mathrm{~m} / \mathrm{s}$ resulting in a Reynolds number range of (0.4-3.5) $\times 10^{5}$. In the simulation the mean velocity was increased up to $14 \mathrm{~m} / \mathrm{s}$ with $1 \mathrm{~m} / \mathrm{s}$ increment. Dominant frequencies and Strouhal numbers are reported from cavitation-free flow (classical von Kármán vortex shedding) up to supercavitation as a function of the free-stream Reynolds number. It was found that between the inception of cavitation and the formation of supercavitation the Strouhal number is not affected by cavitation. The results are in a fairly good agreement with the literature.
\end{abstract}

\section{Keywords}

cavitation - cavitation channel vortex shedding - measurement $\cdot$ CFX simulation $\cdot$ square cylinder

\section{Acknowledgement}

The cavitation channel and instrumentation described in this paper was supported by a grant from National Scientific Research Found (OTKA), Hungary, Project No: 061460.

\section{Ferenc Hegedús}

Department of Hydrodynamic Systems, BME, H-1111 Budapest, Múegyetem rkp 1., Hungary

e-mail: hegedusf@hds.bme.hu

\section{Roland Rákos}

Knorr-Bremse Hungary Ltd., Hungary

\section{László Kullmann}

Department of Hydrodynamic Systems, BME, H-1111 Budapest, Múegyetem rkp 1., Hungary

\section{Introduction}

Cavitation is known as a destructive phenomenon and its avoidance in hydraulic machinery is of essential interest in engineering. The numerical simulation of cavitation is challenging due to the complex physical properties of multiphase flows. Our main aim is to compare the numerical and experimental results obtained on a square cylinder which has the advantage of a simple geometry and well-defined separation points.

Although there are many studies dealing with cavitational flows, e.g. hydrofoils [1], [2], circular cylinder [3], confuserdiffuser test section [4, 5], triangular cylinder and sphere [6], only few studies concern with the simple geometry of the square cylinder, for instance Wienken [7].

The flow around a square cylinder was extensively studied but mostly without cavitation. Although the experiments were usually performed in wind tunnels where cavitation never occurs, the results can be compared to our findings in the cavitation-free regime. Okajima in [8] examined rectangular cylinders with different side ratios $(\mathrm{B} / \mathrm{H}=1$ to 4$)$ in the range of Reynolds numbers $(\mathrm{Re})$ between 70-20000. The Strouhal number for the square cylinder was approximately constant and changed slightly between $0.13-0.14$. The blockage ratio was $0 \%$ (no walls enclosed the model). Sarioglu [9] studied rectangular cylinders with $\mathrm{B} / \mathrm{H}=1$ and 2 at $\mathrm{Re}=13159,53786$ and 99714 . The blockage ratio was $10.94 \%$. In both cases the Strouhal number was constant at 0.12-0.13 and 0.08-0.09, respectively not depending on the Reynolds number. Lyn [10] examined the square cylinder at $\operatorname{Re}=21400$. The results showed good agreement with those of Okajima [8], St $=0.132$. The following papers obtained the same experimental results for square cylinders: Saha [11], Davis [12] and Nakagawa [13]. Davis [12] used relatively low Reynolds numbers (100-1850) but the effect of the blockage ratio was examined. It was clearly seen that with the increase of the blockage ratio $(0 \%, 16.6 \%, 25 \%)$ the Strouhal number $(\sim 0.13, \sim 0.16, \sim 0.17)$ increased too. As for the numerical simulation, the results usually show good agreement with experimental data. Kim [14] performed large eddy simulation for a square cylinder at $\mathrm{Re}=3000$ with blockage ratios $0 \%$ and $20 \%$. The Strouhal numbers were 0.125 and 0.124 , respectively. It is 
worth noting that the Strouhal number in case of a blockage ratio of $20 \%$ should be greater according to Davis [12]. Sohankar [15] also used large eddy simulation but at $\operatorname{Re}=10^{5}$ the blockage ratio was $6 \%$ and the Strouhal number was 0.13 .

Cavitation around a square cylinder dealt with in this paper is suggested to be a benchmark problem for unsteady cavitating CFD calibrations. Its simple geometry allows easy mesh generation with high quality and the sharp corners reduce the uncertainty in predicting the separation point. The present study intends to compare numerical and experimental results; furthermore we compare the results with those found in the literature. The basis of the comparison is the Strouhal number of vortex shedding at different Reynolds numbers. The measurement is based on the pressure signal recorded in the side walls of the test section and on the upper wall of the square cylinder.

\section{Experimental set-up}

The schematic drawing of the cavitation channel of the Department of Hydrodynamic Systems of the Budapest University of Technology and Economics can be seen in Fig. 1 The channel is $4.9 \mathrm{~m}$ high and $5.7 \mathrm{~m}$ long and most of it was built up by $\emptyset 400 \mathrm{~mm}$ and $\emptyset 500 \mathrm{~mm}$ conduits. The $1 \mathrm{~m}$ long test section is made of stainless steel and Plexiglas. In the closed system the water is circulated by an axial pump driven by a $34 \mathrm{~kW}$ electric motor. The revolution speed is controlled by a frequency converter, thus arbitrary revolution speeds can be set in the range of 30-730 rpm. Directly in front of the test section there is a confuser in which the flow velocity increases significantly. The achievable maximum mean velocity is approximately $12 \mathrm{~m} / \mathrm{s}$ depending on the geometry of the test body. The elbow connected to the confuser section includes guide vanes to avoid flow separation. The test section has a rectangular cross section with dimensions of $50 \mathrm{~mm} \times 200 \mathrm{~mm}$. The model with $25 \mathrm{~mm}$ side length is placed $240 \mathrm{~mm}$ after the longitudinal centre of the test section. At the downstream side of the test section a reservoir is located providing constant downstream overpressure which was $\mathrm{H}_{0}=1.28 \mathrm{~m}$ of water column throughout the experiments.

During the measurements five pressure transducers and one ultrasonic flowmeter were used. The arrangement of the pressure taps is shown in Fig. 2. Three pressure transducers were used to determine the vortex shedding frequency after the model, namely, one placed on the top of the model (S1, HBM P6A 10 bar), further one placed in the test section after $12.5 \mathrm{~mm}$ of the trailing edge (S2, HBM P6A 10 bar) and the last one was 625 $\mathrm{mm}$ after the model and $50 \mathrm{~mm}$ above the centre line (S5, HBM P6 10 bar). The pressure difference on the confuser was measured with a mercury-filled single tube manometer, a reversed U-tube manometer containing air above the water and a pressure difference transducer (S3, HBM PD1 1 bar). The pressure difference was used to determine the mean velocity in the test section which is calibrated with the ultrasonic flowmeter placed at the suction tube of the circulating pump (S6). For measuring the absolute pressure level the pressure signal was recorded in front of the model (S4, HBM P6 10 bar). The time average of this pressure time series was used for the reference pressure $\mathrm{p}_{r}$. The measured signals were recorded by a PC through an HBM 840X type A/D converter. The ultrasonic flowmeter was manufactured by FLEXIM, type UMADM5X07-PU1-2EN. The results will be interpreted with the help of dimensionless numbers; namely the Thoma's cavitation number, the Reynolds number and the Strouhal number defined as:

$$
\sigma=2 \frac{p_{r}-p_{v}}{\rho U^{2}}, \quad R e=\frac{U H}{v} \text { and } S t=\frac{f_{H}}{U},
$$

where $\mathrm{p}_{r}$ is the free stream reference pressure, $\mathrm{p}_{v}$ is the vapour pressure (3500 $\mathrm{Pa}), \rho$ is the density $\left(1000 \mathrm{~kg} / \mathrm{m}^{3}\right)$ and $v$ is the kinematic viscosity $\left(0.80110^{-6} \mathrm{~m}^{2} / \mathrm{s}\right)$ of the liquid, $U$ is the mean velocity in the test section (before the obstacles), $H$ is the height of the cylinder and $f$ denotes frequency.

The quantities used in Eqs. (1a-c) contain uncertainties. The pressure measurement includes systematic and random errors of which the first one can be eliminated by calibration. The random error was reduced by taking the average of the measured signals. The signals of $20 \mathrm{~s}$ duration with a $600 \mathrm{~Hz}$ sample rate contain 120000 points. Setting up the significance level to $95 \%$ and assuming a Gaussian distribution the absolute error for the pressure measurement is $10.36 \mathrm{~Pa}$. As for the velocity measurement the ultrasonic flowmeter cannot be calibrated, therefore the absolute error of the mean velocity comes from both the systematic and random error. The class of accuracy of the device is $2 \%$, from which the maximum absolute error of the mean velocity is $0.158 \mathrm{~m} / \mathrm{s}$. The last quantity is the frequency calculated by Fourier transformation of $20 \mathrm{~s}$ long time signals yielding an absolute error of approximately $0.05 \mathrm{~Hz}$. Finally, the relative error of the Thoma, Reynolds and Strouhal number can be calculated as results of indirect measurements from the results above being $2.98 \%, 2.12 \%$ and $2.02 \%$, respectively.

\section{Computational parameters}

In the numerical simulations the ANYSY CFX v10.0 commercial software code was used. For saving computational resources we used a two dimensional $400 \mathrm{~mm}$ high $1200 \mathrm{~mm}$ long domain shown in Fig. 3. The model with $20 \mathrm{~mm}$ side length was placed $200 \mathrm{~mm}$ after the inlet boundary. The inlet boundary condition was constant velocity with normal speed 1 up to $14 \mathrm{~m} / \mathrm{s}$ with $1 \mathrm{~m} / \mathrm{s}$ increment. At the outlet 1 bar static pressure was defined. At the top and bottom of the model there were free slip walls. At the front and rear side of the domain symmetry boundary condition were used leading to two dimensional problems. The structured mesh was prepared in ICEM and contains 113000 hexahedral elements. We also used a coarser mesh but we did not experience significant differences between the results. 


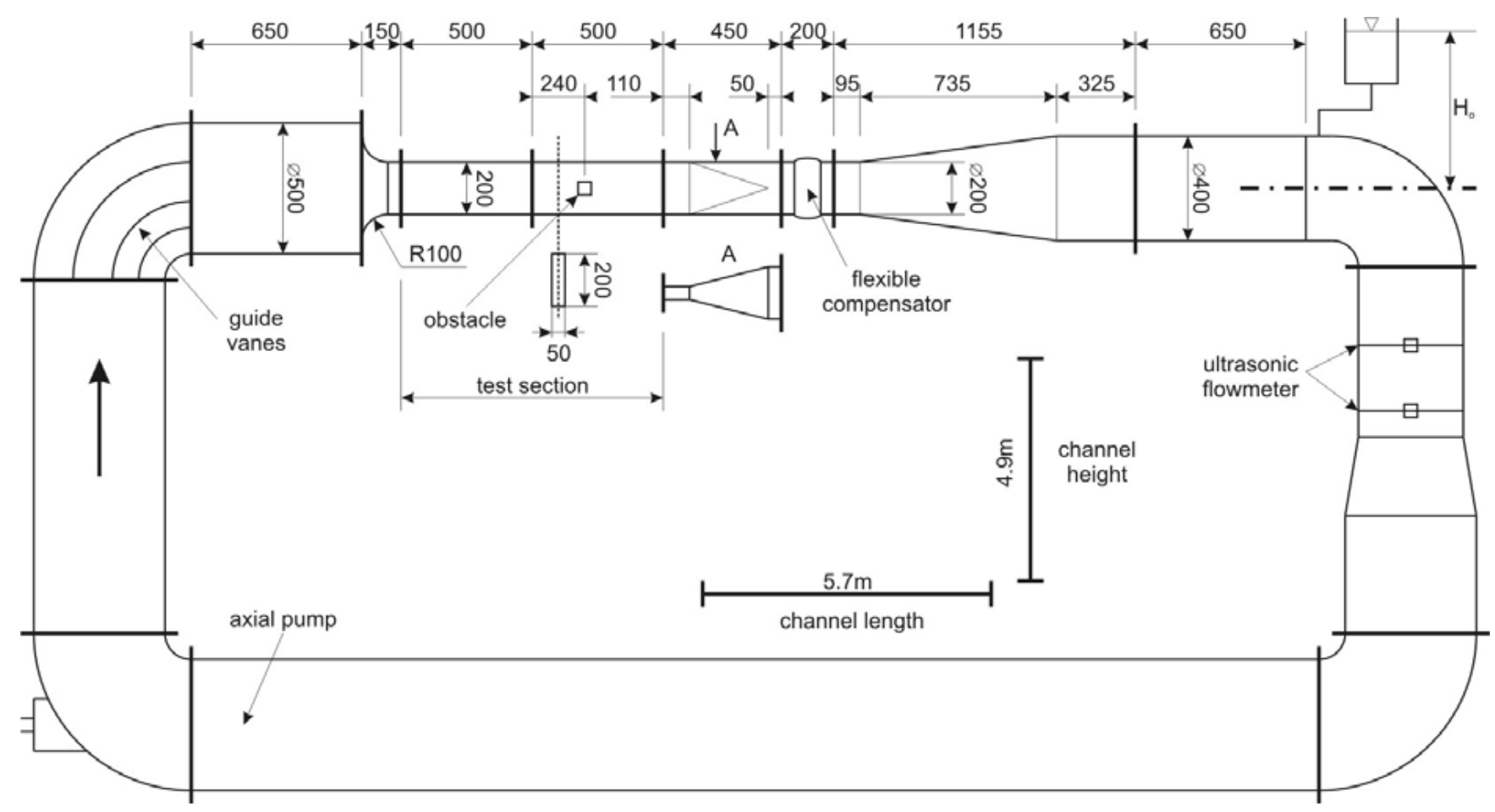

Fig. 1. Geometry of the cavitation channel and the test section.
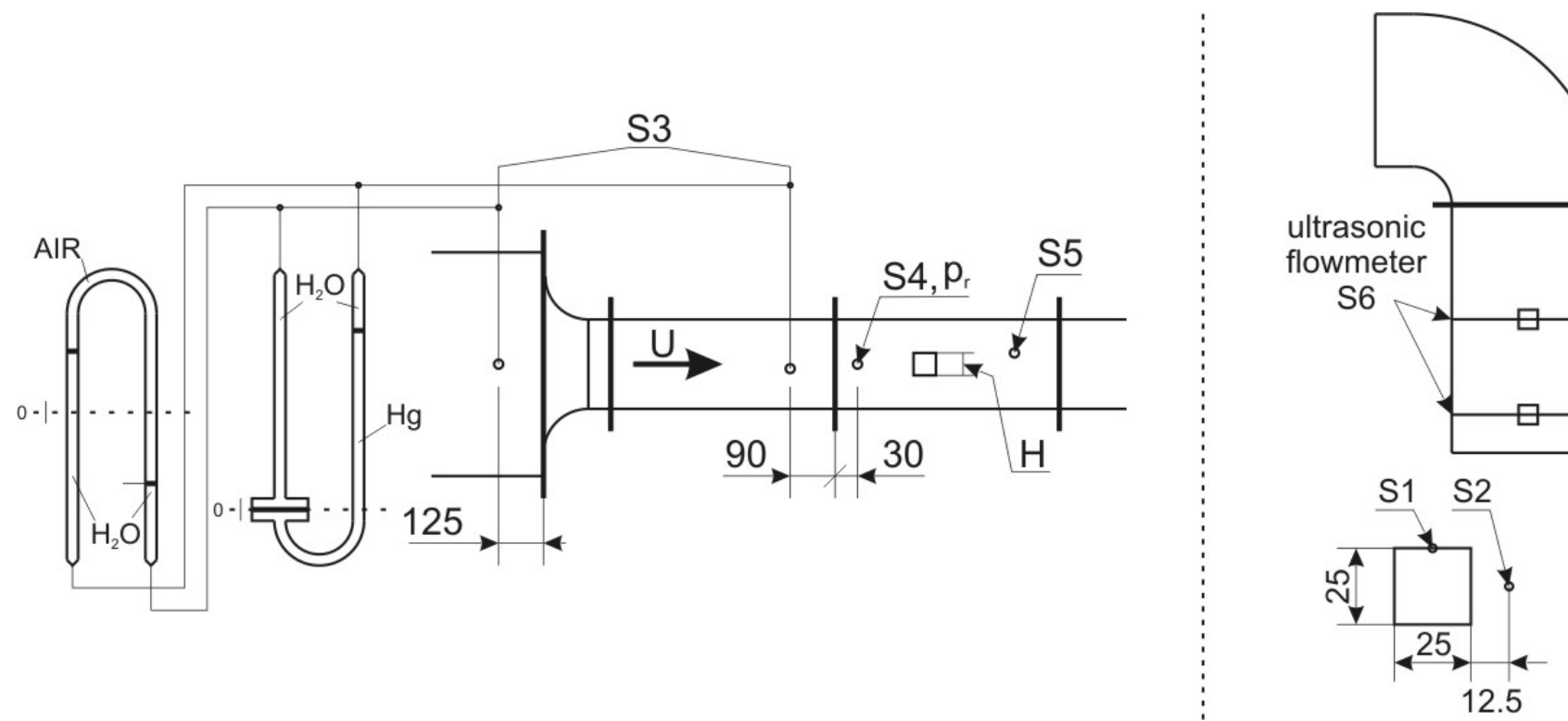

Fig. 2. Sensor arrangement

During the simulation the fluid flow was modelled as homogeneous multiphase flow, that is, all fluids share the same velocity fields, as well as other relevant fields such as temperature, turbulence, etc. The pressure field is also shared by all fluids. In our simulations water and water vapour phases were defined, both phases were incompressible. The heat transfer and thermal radiation were not modelled. The turbulence model was SAS SST. It is worth noting that the $\mathrm{k}-\varepsilon$ turbulence model overdissipates the system therefore in this case there is no vortex shedding behind the model. The mass transfer was modelled by the simplified Rayleigh-Plesset model which is built into CFX.

The advection scheme (spatial discretization) and the tran-

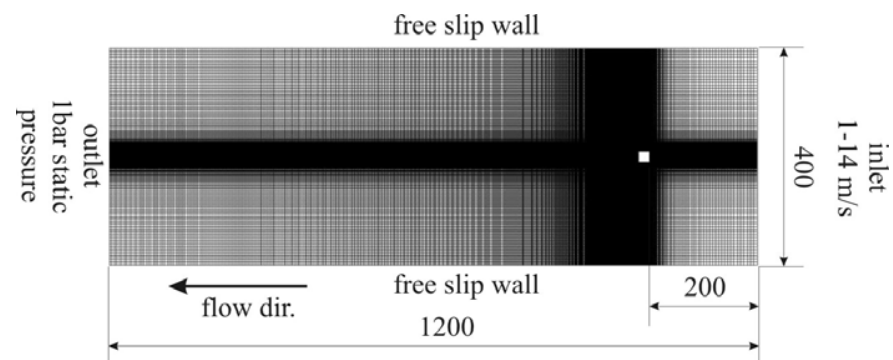

Fig. 3. The two-dimensional model of the multiphase flow

sient scheme were high resolution and second order backward Euler scheme, respectively. The constant time step was $0.001 \mathrm{~s}$. 
Tab. 1. Pictures about the multiphase flow at different time instants. Upper row: measurement. Lower row: simulation. The pictures correspond to the
Reynolds numbers marked by thick lines in Fig. 6
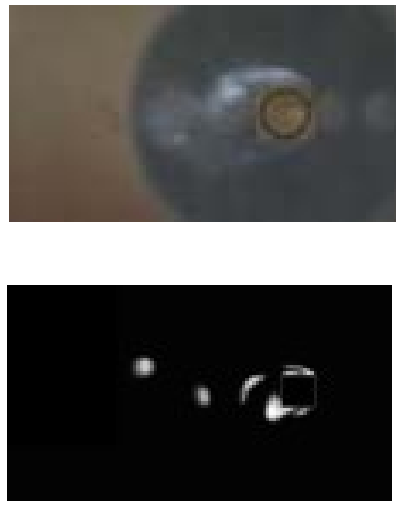
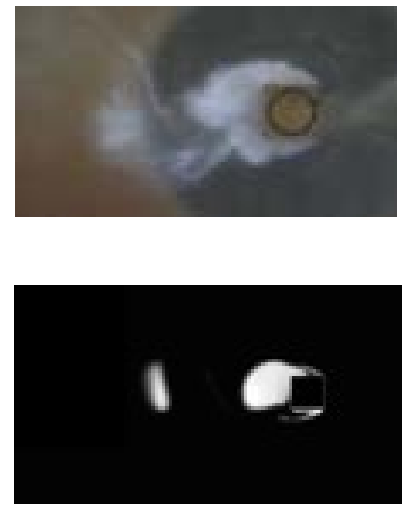
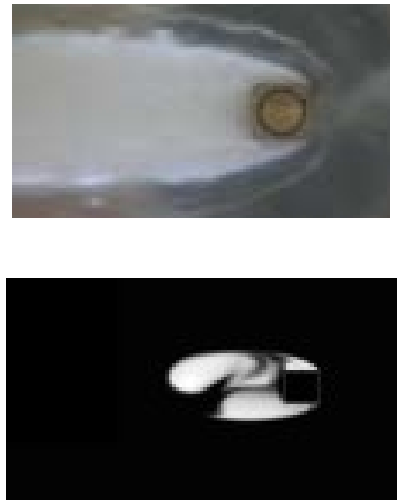

The convergence criterion was $10^{-4}$ for the root mean square of the residuals. Pressure and velocity time series were recorded only in a few monitor points shown if Fig. 4 .

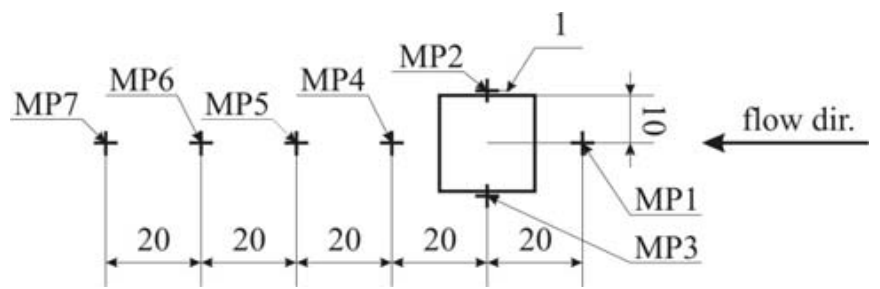

Fig. 4. Monitor points distribution

\section{Results}

Fig. 5 shows typical waterfall diagrams of the pressure signals measured by sensor S1 (upper horizontal wall of the cylinder, see Fig. 2) and calculated by numerical simulation at monitor point MP2. Each spectrum was normalized by its maximum. In case of the measurement for low and medium flow velocities, we experienced a single dominant frequency and its linear increase with flow velocity, corresponding to von Kármán vortex shedding frequency. The first harmonic with double frequency is also clearly seen in this range. Between approx. 6 and 8 $\mathrm{m} / \mathrm{s}$, there is an intermediate range with wide frequency content where the appearing frequencies are beneath the dominant one. Finally, above approximately $8.5 \mathrm{~m} / \mathrm{s}$, only a very low-frequency component is present corresponding to the appearance of a massive cavitating cloud (supercavitation). The measurements were also performed at different sample rates $(600 \mathrm{~Hz}, 1200 \mathrm{~Hz}, 2400$ $\mathrm{Hz}$ ) but the higher sample rates did not yield any additional information, therefore the sample rate $600 \mathrm{~Hz}$ was used in later experiments. As for the numerical simulation the spectra are very noisy but the linear relationship between the characteristic frequencies (first peaks) and mean velocity can be seen below approximately $8 \mathrm{~m} / \mathrm{s}$. The incidence of cavitation occurs at $7 \mathrm{~m} / \mathrm{s}$ but the relatively small vapour bubbles have no effect on the frequency of vortex shedding. Above this mean velocity the spectra show white noise characteristics and the definite first peak disappears due to the larger cavitational zone.

Our main interest is the relationship between the Strouhal number (dimensionless frequency) and Reynolds number (dimensionless velocity). In order to get a better picture about the relation, the same data set is represented again in Fig. 6 . in which the scatter plots depict the peaks above $30 \%$ and $60 \%$ of the actual spectrum in case of the measurement and simulation respectively. As we have expected from the literature, the Strouhal number is nearly constant in a wide range of Reynolds numbers. The average Strouhal number is approximately 0.18 in measurement and 0.1 in case of the simulation. The difference between these values is presumably due to the different blockage ratios $(12.5 \%$ and $5 \%)$. The inception of cavitation occurs at slightly lower Reynolds and Thoma numbers in simulation than in the measurement. Comparing the results with Wienken [7], in which the cavitation onset is at $\operatorname{Re}=10^{5}$ and $\sigma_{i n c}=5.75$, shows fairly good agreement.

Table 1 represents three photos of the measurement and three pictures of the simulation at different Reynolds numbers at a given instant. These pictures belong to the Reynolds numbers marked by thick lines with the labels left, centre and right in Fig. 6. In case of the simulation (lower row) the white bubbles represent the vapour phase. At the highest Reynolds number the cavitation zone length is much larger in the measurement than in the simulation.

Table 2 includes a comparison of the results of our research with those of other papers. Most of these papers deal with an infinite flow domain, namely the blockage ratio is $0 \%$. Although Davis[12] has very low Reynolds numbers, his result shows a good agreement with the present study at the nearly same blockage ratio. There are some discrepancies between the results of the experimental and numerical studies. It seems that in the numerical investigations the blockage ratio has no effect on the Strouhal number, see [14]. Furthermore in our simulation the 

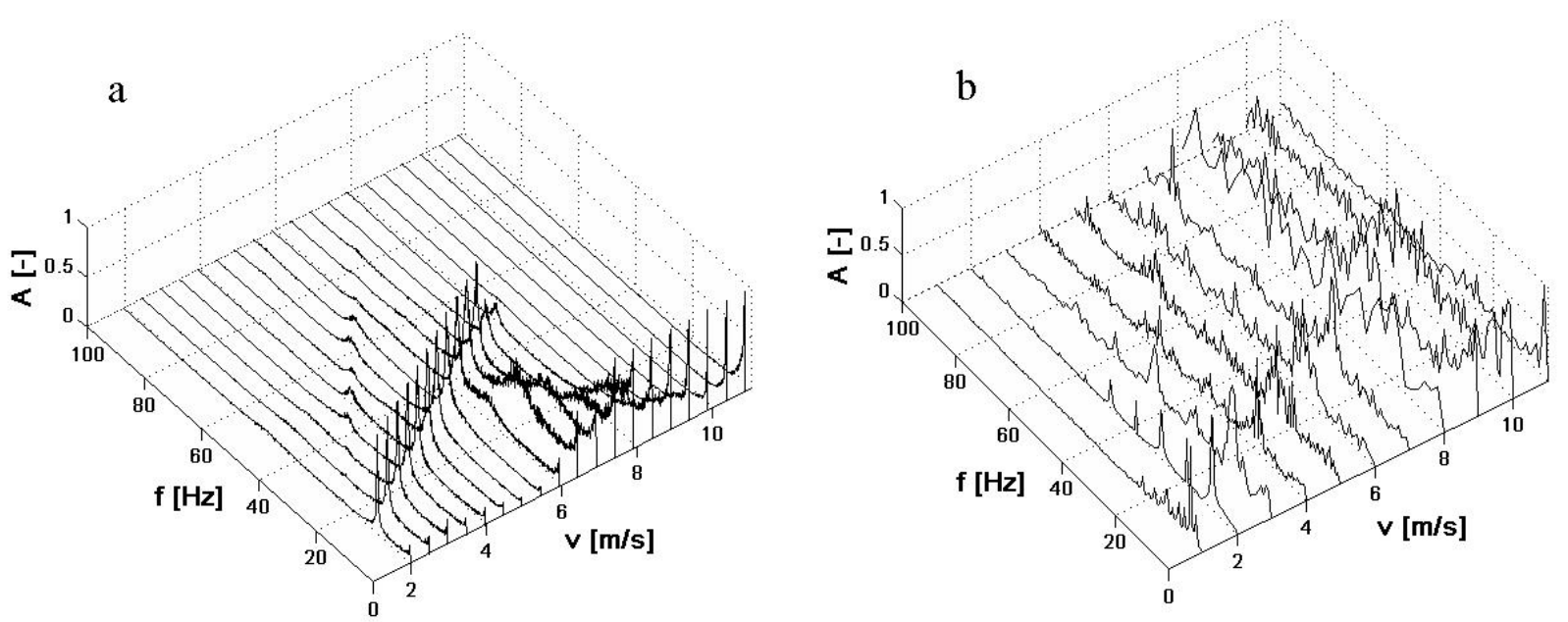

Fig. 5. Waterfall diagrams; a: measurement, b: simulation
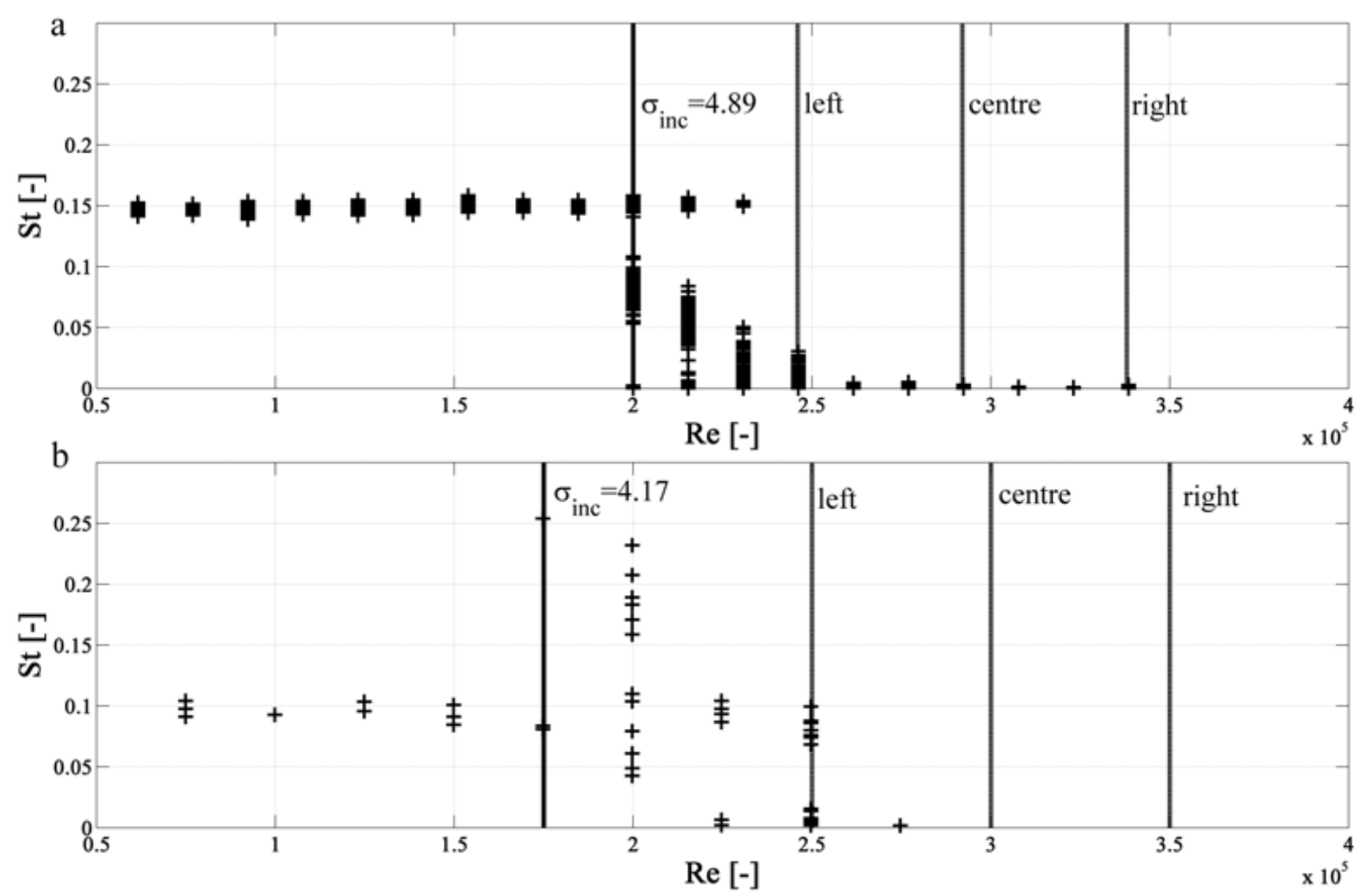

Fig. 6. Strouhal number versus Reynolds number; a: measurement, b: simulation

Strouhal number is even lower than in other numerical papers which is most likely due to the different turbulence model.

\section{Conclusion}

Unsteady cavitating vortex shedding was studied at high Reynolds numbers $\left(>5 \times 10^{4}\right.$ ) behind square cylinders. The side lengths and blockage ratios were $25 \mathrm{~mm}, 20 \mathrm{~mm}$ and $12.5 \%$, $5 \%$ in the experimental and numerical study, respectively. The power spectra and dominant frequencies of the pressure signals in the wake were presented as a function of the Reynolds number.

A qualitative correspondence was found with the literature in the sense that although we were unable to find measurements and simulations at such high Reynolds numbers, our Strouhal numbers provide a natural extension of the existing data: several researchers report that if the Reynolds number exceeds $10^{4}$, the dominant Strouhal number seems to stabilize at around 0.130.18 depending on the blockage ratio. We also found that unless the state of supercavitation is reached, cavitation does not affect the Strouhal number.

Our numerical and experimental results are somewhat inconsistent with the literature which became evident in the relatively low value of the average Strouhal number. 
Tab. 2. Comparison of the experimental and numerical results.

\begin{tabular}{|c|c|c|c|c|}
\hline Author & Type & St & $\mathbf{R e}$ & $\begin{array}{l}\text { blockag } \\
\text { ratio }\end{array}$ \\
\hline Okajima [8] & Experimental & 0.13 & $70-20000$ & $0 \%$ \\
\hline Lyn 10 & Experimental & 0.132 & 21400 & $0 \%$ \\
\hline \multirow[t]{2}{*}{ Saha[11]. } & Experimental & 0.144 & 8700 & $0 \%$ \\
\hline & Experimental & 0.142 & 17625 & $0 \%$ \\
\hline \multirow[t]{3}{*}{ Davis 12} & Experimental & 0.13 & $100-1850$ & $0 \%$ \\
\hline & Experimental & 0.16 & $100-1850$ & $16.6 \%$ \\
\hline & Experimental & 0.17 & $100-1850$ & $25 \%$ \\
\hline Nakagawa 13 & Experimental & 0.13 & 3000 & $20 \%$ \\
\hline \multirow[t]{2}{*}{$\operatorname{Kim} 14$} & Numerical & 0.124 & 3000 & $20 \%$ \\
\hline & Numerical & 0.125 & 3000 & $0 \%$ \\
\hline Sohankar 15 & Numerical & 0.13 & 100000 & $6 \%$ \\
\hline Present & Numerical & 0.1 & $0.510^{4}-3.510^{5}$ & $5 \%$ \\
\hline Present & Experimental & 0.15 & $0.510^{4}-3.510^{5}$ & $12.5 \%$ \\
\hline
\end{tabular}

\section{References}

1 Foeth E J, van Doorne C W H, van Terwisga T, Wieneke B, Time resolved PIV and flow visualization of $3 D$ sheet cavitation, Experiments in Fluids 40 (2006), 503-513, DOI 10.1007/s00348-005-0082-9.

2 Lohrberg H, Stoffel B, Fortes-Patella R, Coutier-Delgosha O, Rebound $\mathbf{J} \mathbf{L}$, Numerical and experimental investigations on the cavitating flow in a cascade of hydrofoils, Experiments in Fluids 33 (2002), 578-586, DOI 10.1007/s00348-002-0508-6.

3 Saito Y, Sato K, Cavitation bubble collapse and impact in the wake of a circular cylinder, Fifth International Symposium on Cavitation (CAV2003), 2003.

4 Stutz B, Legoupil S, X-ray measurements within unsteady cavitation, Experiments in Fluids 35 (2003), 130-138, DOI 10.1007/s00348-003-0622-0.

5 Stutz B, Rebound J L, Measurements within unsteady cavitation, Experiments in Fluids 29 (2000), 545-552, DOI 10.1007/s003480000122.

6 Schmidt S J, Sezal I H, Schnerr G H, Thalhamer M, Numerical analysis of shock dynamics for detection of erosion sensitive areas in complex 3-D flows, Cavitation: Turbo-machinery \& Medical Applications WIMRC FORUM, 2008.

7 Wienken W, Stiller J, Keller A, A method to predict cavitation inception using large-eddy simulation and its application to the flow past a square cylinder, Journal of Fluids Engineering 128 (2006), no. 2, 316-326, DOI 10.1115/1.2170132.

8 Okajima A, Strouhal numbers of rectangular cylinders, Journal of Fluid Mechanics 123 (1982), 379-398, DOI 10.1017/S0022112082003115.

9 Sarioglu M, Yavuz T, Vortex shedding from circular and rectangular cylinders placed horizontally in a turbulent flow, Turkish Journal of Engineering \& Environmental Sciences 24 (2000), 217-228.

10 Lyn D A, Einav S, Rodi W, Park J H, A laser-Doppler velocimetry study of ensemble-averaged characteristics of the turbulent near wake of a square cylinder, Journal of Fluid Mechanics 304 (1995), 285-319, DOI 10.1017/S0022112095004435.

11 Saha A K, Muralidhar K, Biswas G, Experimental study of flow past a square cylinder at high Reynolds number, Experiments in Fluids 29 (2000), 553-563, DOI 10.1007/s003480000123.

12 Davis R W, Moore E F, and Purtell L P, A numerical-experimental study of confined flow around rectangular cylinders, Physics of Fluids 27 (1984), no. 1, 46-59, DOI 10.1063/1.864486.

13 Nakagawa S, Nitta K, Senda M, An experimental study on unsteady turbulent near wake of a rectangular cylinder in channel flow, Experiments in Fluids 27 (1999), no. 3, 284-294, DOI 10.1007/s003480050353.

14 Kim D H, Yang K S, Senda M, Large eddy simulation of turbulent flow past a square cylinder confined in a channel, Computers \& Fluids 33 (2004), 81-96, DOI 10.1016/S0045-7930(03)00040-9.

15 Sohankar A, Large eddy simulation of flow past rectangular-section cylinders: Side ratio effects, Journal of Wind Engineering 96 (2008), 640-655, DOI 10.1016/j.jweia.2008.02.009. 